\title{
Cerebral air embolism and
} recipient following the removal of the pulmonary artery catheter from the central venous access device

\section{-a case report-}

\author{
Sun-Key Kim, In-Gu Jun, Dong-Min Jang, Jinwook Lim, Gyu-Sam Hwang, \\ and Young-Kug Kim \\ Department of Anesthesiology and Pain Medicine, Asan Medical Center, University of Ulsan College of Medicine, \\ Seoul, Korea
}

Cerebral air embolism is a rare but potentially life-threatening complication. We experienced a living-donor liver transplant recipient who presented with unexpected cerebral air embolism and transient neurologic abnormalities that subsequently developed just after the removal of the pulmonary artery catheter from the central venous access device. One day after the initial event, the patient's neurologic status gradually improved. The patient was discharged 30 days after liver transplantation without neurologic sequelae.

Key Words: Air embolism, Central venous catheters, Liver transplantation.

Pulmonary artery catheters are used to monitor cardiac output, right ventricular end-diastolic volume, pulmonary artery pressure, pulmonary capillary wedge pressure, mixed venous oxygen saturation, and right ventricular ejection fraction in

Corresponding author: Young-Kug Kim, M.D., Ph.D.

Department of Anesthesiology and Pain Medicine, Asan Medical Center, University of Ulsan College of Medicine, 88, Olympic-ro 43gil, Songpa-gu, Seoul 05505, Korea

Tel: 82-2-3010-5976, Fax: 82-2-3010-6790

E-mail: kyk@amc.seoul.kr

Received: July 18, 2014

Revised: July 28, 2014.

Accepted: August 7, 2014.

Korean J Anesthesiol 2016 February 69(1): 80-83

http://dx.doi.org/10.4097/kjae.2016.69.1.80 critically ill patients [1]. Most especially, liver transplant recipients with severe liver disease demonstrate specific characteristics such as low systemic vascular resistance and high cardiac output [2]. Furthermore, severe hemodynamic fluctuation can develop after clamping the inferior vena cava, reperfusion of new liver graft, severe hemorrhage, and acid-base disturbance during liver transplantation. Therefore, pulmonary artery catheterization may be preferred for hemodynamic monitoring during liver transplantation.

However, pulmonary artery catheterization can result in adverse effects. In particular, paradoxical cerebral air embolism which is a rare but potentially life-threatening complication can develop during the insertion or removal of the pulmonary artery catheter. Interestingly, the inflow route of air can be the lumen or rubber valve of the central access device as well as the lumen of pulmonary artery catheter.

(c) This is an open-access article distributed under the terms of the Creative Commons Attribution Non-Commercial License (http://creativecommons.org/ licenses/by-nc/4.0/), which permits unrestricted non-commercial use, distribution, and reproduction in any medium, provided the original work is properly cited. 
We experienced a living-donor liver transplant recipient who developed cerebral air embolism and subsequent neurologic abnormalities just after the removal of the pulmonary artery catheter from the central venous access device. Through this case study, we discuss the mechanisms, diagnosis, treatment, and prevention of cerebral air embolism and review the available literature.

\section{Case Report}

A 61-year-old man (body weight $=69.5 \mathrm{~kg}$; height $=166 \mathrm{~cm}$ ) was scheduled to receive living-donor liver transplantation due to hepatitis B virus-related liver cirrhosis and hepatocellular carcinoma. The patient had developed lung cancer 16 years prior, which was treated with chemotherapy and radiotherapy, and was in complete remission. Preoperative chest X-ray revealed patterns indicative of peripheral interstitial lung disease in the left upper and left middle lobe due to radiotherapy, and pulmonary function testing revealed a moderate restrictive pattern. However, the patient developed no respiratory symptoms, such as dyspnea on exertion, cough, or sputum, and arterial blood gas analysis provided results within the normal range. Transthoracic echocardiography results were also normal, and no intracardiac shunting was noted on color flow imaging. Magnetic resonance imaging (MRI) of the brain was performed as part of our routine preoperative evaluation, and the results were normal. The patient's Child-Pugh and Model for End-Stage Liver Disease scores were 8 and 20, respectively.

The patient entered the operating room without receiving preoperative medications. Monitoring was performed using electrocardiography, noninvasive blood pressure monitoring, and pulse oximetry. The radial artery was cannulated for contin-

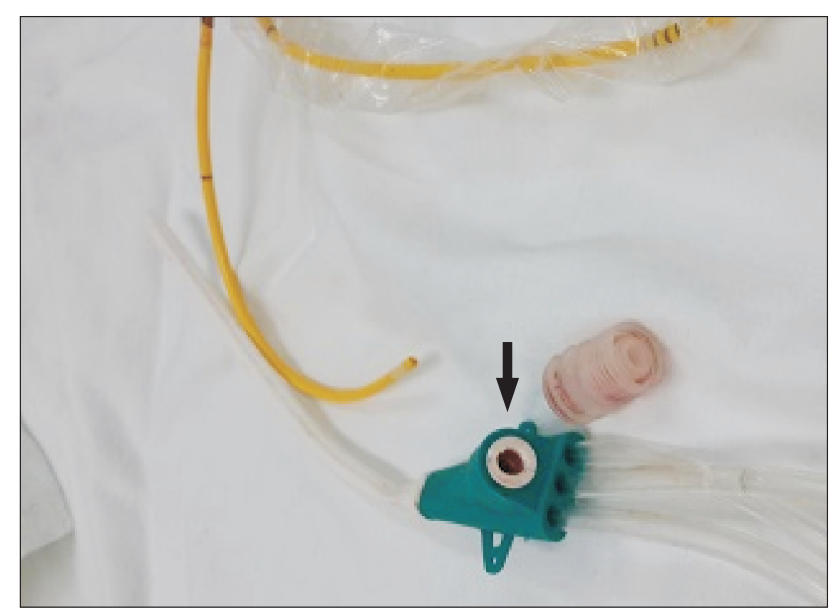

Fig. 1. The central venous access device. The black arrow indicates the insertion site of the pulmonary artery catheter. The yellow catheter is the pulmonary artery catheter. uous arterial blood pressure monitoring. The patient's initial systolic/diastolic arterial blood pressure was 115/60 $\mathrm{mmHg}$, heart rate was 80 beats/min, and oxygen saturation was $97 \%$ on pulse oximetry. Anesthesia was induced by using $5 \mathrm{mg}$ midazolam, $300 \mathrm{mg}$ thiopental sodium, $100 \mu \mathrm{g}$ fentanyl, $10 \mathrm{mg}$ vecuronium, and 2 vol\% sevoflurane and maintained with $0.7 \mathrm{~L} / \mathrm{min} \mathrm{O}_{2}, 1.3$ $\mathrm{L} / \mathrm{min}$ air, $1.0-1.5$ vol\% sevoflurane, and 3-5 $\mu \mathrm{g} / \mathrm{kg} / \mathrm{h}$ fentanyl. A central venous access device (AVA HF; Edwards Lifesciences, Irvine, CA, USA) was inserted into the right internal jugular vein under ultrasound guidance (M-Turbo; Fujifilm Sonosite, Bothell, WA, USA). A pulmonary artery catheter (Swan-Ganz CCOmbo; Edwards Lifesciences) was inserted through the central venous access device, and then liver transplantation was performed. Ten hours after anesthesia induction, liver graft reperfusion was performed and no signs of postreperfusion syndrome developed. The total anesthesia time was 15 hours. Three units of filtered and irradiated red blood cells, 4 units of fresh frozen plasma, and 10 units of cryoprecipitate were transfused during surgery.

After surgery, the patient was transferred to the surgical intensive care unit. Noradrenaline was administered at a rate of $0.15 \mu \mathrm{g} / \mathrm{kg} / \mathrm{min}$ due to low diastolic arterial blood pressure, and the endotracheal tube was maintained. Eight hours after surgery, the patient could keep his eyes open as recommended, but still tended to want to sleep. The patient was placed in the semiFowler's position (back raised about $30^{\circ}$ ) and was ventilated with pressure-control mode with an inspiratory oxygen fraction $\left(\mathrm{FIO}_{2}\right)$ to $50 \%$. The pulmonary artery catheter was removed from the central venous access device. At that time, the patient suddenly inhaled deeply and got up from the bed. He started coughing, and blood and air suddenly spouted from the rubber valve of the central venous access device (Fig. 1). Within $90 \mathrm{sec}-$

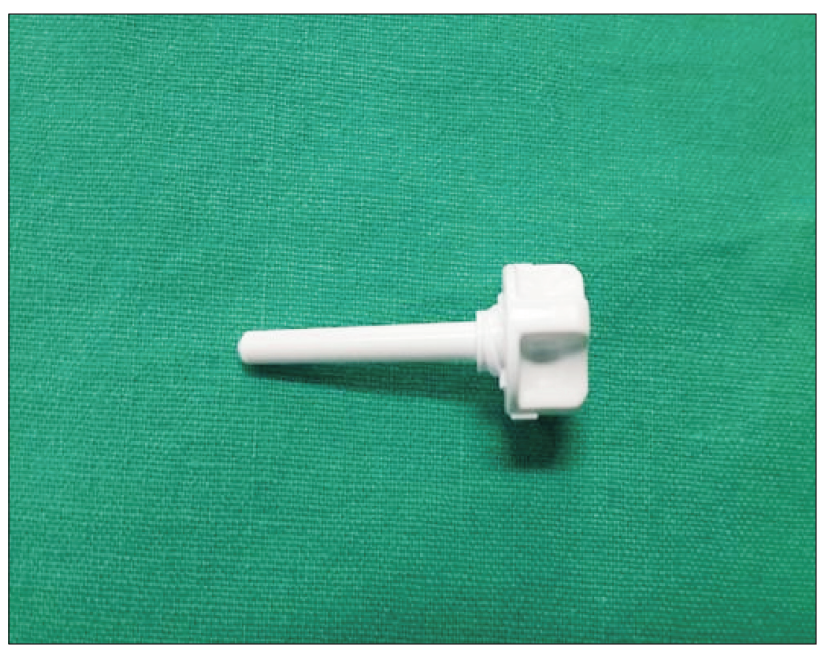

Fig. 2. The obturator plug of the central venous access device. 
onds, the patient's pulse oximetry oxygen saturation decreased to $88 \%$ and he became drowsy. His arterial blood pressure was $110 / 55 \mathrm{mmHg}$, and his heart rate was 87 beats/min. The patient was placed in the Trendelenburg and the left lateral decubitus position, which is known as Durant's maneuver. We attempted to aspirate air from the central venous access device, but no air bubbles were seen in the syringe and the rubber valve site was closed with an obturator plug (Fig. 2). The ventilator was reset to $100 \% \mathrm{FIO}_{2}$. A neurologist performed a neurologic examination, and the patient had left-sided paralysis and was stuporous. The patient's bispectral index was 70. At this time, emergency computed tomography (CT) of the brain was performed, and the results only indicated mild brain atrophy. Chest X-ray showed no changes in comparison with the preoperative state, except mild linear subsegmental atelectasis in the right middle lung zone.

Three hours later, MRI with diffusion-weighted imaging was performed, and there were very tiny, cortical, high signal, dot-like lesions in both hemispheres, indicating microemboli (Fig. 3). However, the neurologists did not recommend hyperbaric oxygen therapy because the lesion was very small. The central venous access device was removed when the patient was in the Trendelenburg position, and pressure was maintained by hand for 10 minutes at the insertion site of the central venous access device. The dressing consisted of gauze and Tegaderm.

One day after the initial event, the patient's neurologic status was gradually improved. He could open his eyes in response to verbal commands. The patient had normal mental status by 3 days after the insult had occurred. On the sixth day postoperation, the patient was extubated and transferred to the general

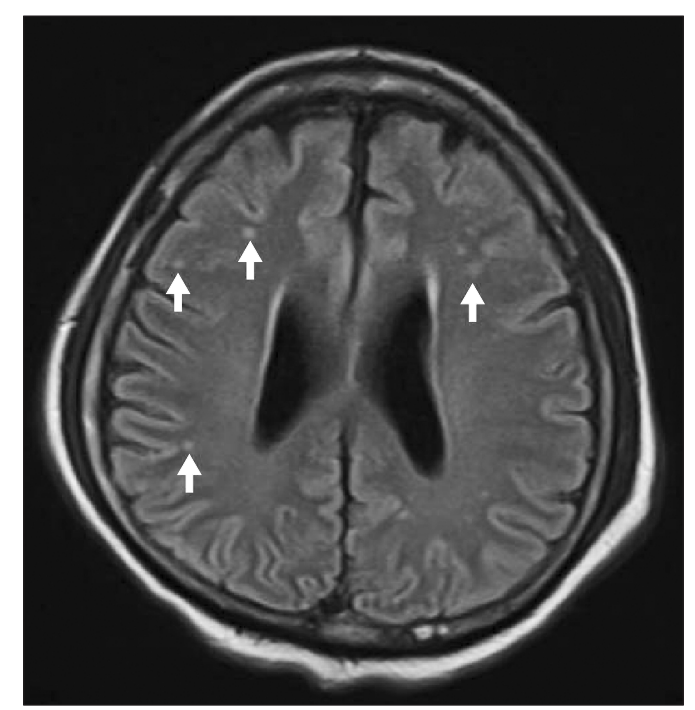

Fig. 3. Brain magnetic resonance imaging of the study patient showing very tiny, cortical, high signal lesions in both cerebral hemispheres. The arrows indicate white dot-like lesions, which are suggestive of microemboli. ward. At that time, his motor weakness had improved, and he could walk with maximum assistance. Active physical therapy was performed by the rehabilitation team. The patient was discharged 30 days after surgery with no neurologic sequelae.

\section{Discussion}

Our present report describes an unexpected case of cerebral air embolism and subsequent transient neurologic abnormalities that developed following the removal of a pulmonary artery catheter from the central venous access in a liver transplant recipient. The introduction of air into the venous or arterial system can cause cerebral air embolism and subsequently various neurologic deficits. Cerebral air embolism is thought to occur via two mechanisms. One mechanism is that paradoxical embolism occurs in the presence of any right-to-left shunting, including patent foramen ovale and pulmonary arteriovenous shunting $[3,4]$. If there is a patent foramen ovale, elevated pulmonary artery pressure due to venous air embolism may result in elevated right atrial pressure. In this situation, right atrial pressure may exceed left atrial pressure, thereby enabling a bubble to pass through the patent foramen ovale [5]. In addition, hepatopulmonary syndrome, which is also a condition that results in right-to-left shunting, is found in $15 \%-20 \%$ of patients with liver cirrhosis [6]. Despite preoperative echocardiography, right to-left or transpulmonary shunting cannot be ruled out in our current case because pulmonary angiography and agitated saline testing with echocardiography were not included. The other possible mechanism is retrograde venous cerebral embolism via the venous system, which depends on air bubble size and local blood flow velocity $[7,8]$. If the diameter of the venous lumen is larger than that of the air bubble, air bubbles entered into superior vena cava via the central venous access device and may rise retrograde against blood flow to the brain [7]. The retrograde flow of air bubbles occurs at a rate of $0.2 \mathrm{~L} / \mathrm{min}$ when the neck is vertically positioned $>45^{\circ}[8]$.

The risk factors for venous air embolism from a central venous catheter include a sitting position during insertion, disconnection and removal, low central venous pressure, deep inspiration by the patient, failure to occlude the needle hub, disconnection of the catheter from the intravenous line, fracture or laceration of the catheter, failure to use occlusive dressing after removal of the catheter, and the use of a large catheter [9]. The most important problem in our patient was inappropriate positioning and the disuse of the obturator plug of the central venous access device immediately after removal of the pulmonary artery catheter. Moreover, the patient started to deeply and abruptly inspirate, leading to low central venous pressure. Importantly, in order to prevent cerebral air embolism, the pulmonary artery catheter should be cautiously removed during ex- 
piration while the patient is in the Trendelenburg position. Also, after removing the pulmonary artery catheter, the rubber valve must be occluded with the obturator plug in order to minimize the risk of air embolism.

The diagnosis of cerebral air embolism depends on the patient's medical history and clinical suspicion. Neurological symptoms are nonspecific, but include dizziness, paresthesia, convulsions, paralysis, headache, and visual disturbances [3]. Unenhanced CT scans of the brain can detect cerebral air embolism early in the disease course [10]. In addition, Caulfield et al. [11] have reported the MRI characteristics of two cases of cerebral embolism, including multiple areas of restricted diffusion along the cortical grey matter in a gyriform pattern that involved both cerebral hemispheres. However, no current imaging technique alone has demonstrated sufficient accuracy for diagnosing cerebral air embolism. Therefore, clinical evaluation is still preferred. In our current patient, neurologic symptoms developed immediately after the removal of the pulmonary artery catheter, and blood and air were observed through the central venous access device, which allowed instant diagnosis. Furthermore, we used brain CT and MRI to accurately diagnose and treat cerebral air embolism.

The best treatment for a cerebral air embolism is to identify the source of air entry and prevent further inflow [12]. Also, an immediate change to the patient's position using Durant's maneuver, administering oxygen therapy, increasing the venous pressure via fluid intervention, and providing immediate critical care support are needed. Hyperbaric oxygen therapy is recommended, especially within the first 6 hours after embolism [13]. Hyperbaric oxygen therapy diminishes the volume of the intravascular air bubbles by increasing the ambient pressure and oxygen tension in the blood, which reduces the size of any air bubbles $[3,14]$. In our current case, the air embolism was very tiny and the neurologist did not therefore recommend hyperbaric oxygen therapy. The general guidelines for preventing air embolism, such as the Trendelenburg position, occlusion of the needle hub, and providing occlusive dressing after the removal of a catheter, should be strictly performed during central venous access device insertion and removal [15]. In addition, removing the pulmonary artery or central venous catheter should be synchronized with active exhalation in cooperative patients [4].

In conclusion, cerebral air embolism is a rare but very serious complication. It is thus very important for clinicians to understand the conditions that can increase the risk of cerebral air embolism. Prompt diagnosis and treatment are necessary if the clinical symptoms of this condition develop.

\section{References}

1. Nelson LD. The new pulmonary arterial catheters. Right ventricular ejection fraction and continuous cardiac output. Crit Care Clin 1996; 12: 795-818.

2. Moller S, Henriksen JH. Cardiopulmonary complications in chronic liver disease. World J Gastroenterol 2006; 12: 526-38.

3. van Hulst RA, Klein J, Lachmann B. Gas embolism: pathophysiology and treatment. Clin Physiol Funct Imaging 2003; $23: 237-46$.

4. Mirski MA, Lele AV, Fitzsimmons L, Toung TJ. Diagnosis and treatment of vascular air embolism. Anesthesiology 2007; $106:$ 164-77.

5. Muth CM, Shank ES. Gas embolism. N Engl J Med 2000; 342: 476-82.

6. Palma DT, Fallon MB. The hepatopulmonary syndrome. J Hepatol 2006; 45: 617-25.

7. Schlimp CJ, Loimer T, Rieger M, Lederer W, Schmidts MB. The potential of venous air embolism ascending retrograde to the brain. J Forensic Sci 2005; 50: 906-9.

8. Fracasso T, Karger B, Schmidt PF, Reinbold WD, Pfeiffer H. Retrograde venous cerebral air embolism from disconnected central venous catheter: an experimental model. J Forensic Sci 2011; 56 Suppl 1: S101-4.

9. Pronovost PJ, Wu AW, Sexton JB. Acute decompensation after removing a central line: practical approaches to increasing safety in the intensive care unit. Ann Intern Med 2004; 140: 1025-33.

10. Valentino R, Hilbert G, Vargas F, Gruson D. Computed tomographic scan of massive cerebral air embolism. Lancet 2003; $361: 1848$.

11. Caulfield AF, Lansberg MG, Marks MP, Albers GW, Wijman CA. MRI characteristics of cerebral air embolism from a venous source. Neurology 2006; 66: 945-6.

12. Han SS, Kim SS, Hong HP, Lee SY, Lee SJ, Lee BK. Massive paradoxical air embolism in brain occurring after central venous catheterization: a case report. J Korean Med Sci 2010; 25: 1536-8.

13. Blanc P, Boussuges A, Henriette K, Sainty JM, Deleflie M. Iatrogenic cerebral air embolism: importance of an early hyperbaric oxygenation. Intensive Care Med 2002; 28: 559-63.

14. Jørgensen TB, Sørensen AM, Jansen EC. Iatrogenic systemic air embolism treated with hyperbaric oxygen therapy. Acta Anaesthesiol Scand 2008; 52: 566-8.

15. Heckmann JG, Lang CJ, Kindler K, Huk W, Erbguth FJ, Neundörfer B. Neurologic manifestations of cerebral air embolism as a complication of central venous catheterization. Crit Care Med 2000; 28: 1621-5. 